\title{
Topical polyphenol treatment of sacrococcygeal pilonidal sinus disease: use of ultrasonography to evaluate response to treatment - clinical case series study
}

\author{
Hasan Mete Aksoy ${ }^{1}$, Berna Aksoy², Ezgi Ozkur ${ }^{3}$, Emel Calikoglu ${ }^{4}$ \\ 'Department of Plastic and Reconstructive Surgery, Faculty of Medicine, Bahcesehir University, Istanbul, Turkey \\ ${ }^{2}$ Department of Dermatology, Faculty of Medicine, Bahcesehir University, Istanbul, Turkey \\ ${ }^{3}$ Department of Dermatology, Health Sciences University, Sisli Etfal Training and Research Hospital, Istanbul, Turkey \\ ${ }^{4}$ Department of Dermatology, Faculty of Medicine, Aksaray University, Aksaray, Turkey
}

Adv Dermatol Allergol 2019; XXXVI (4): 431-437 DOI: https://doi.org/10.5114/ada.2018.77255

\begin{abstract}
Introduction: Sacrococcygeal pilonidal sinus disease (SPSD) is a common disorder and encountered more frequently in hairy young males after puberty.

Aim: To assess effectiveness of topical polyphenol treatment of SPSD by using physical examination and ultrasonography (USG).

Material and methods: Fourteen patients with SPSD diagnosis were treated by natural polyphenol products and were retrospectively included in this study. All of the patients were assessed by superficial ultrasonography before and after treatment. Demographic and clinical information of the patients was obtained from medical records. Results: Patients were aged 18-45 years and the median disease duration was 2 years. The most common findings in physical examination were sinus openings and subcutaneous nodules and the most common ultrasonographic findings were abscess/cyst formation and presence of fistula at the pre-treatment visit. All of the patients applied topical polyphenols for a median period of 3 months. Topical polyphenol treatment was effective in $92.9 \%$ of patients. Follow-up examinations at 18.3 months post-treatment revealed that 12 (85.7\%) patients were free of disease. Control physical examination and ultrasonographic assessment were completely normal in 11 (78.6\%) patients. Conclusions: Topical polyphenol therapy is a promising alternative treatment for SPSD and its effectiveness can be objectively evaluated by ultrasonography. It is advised that topical polyphenols should be tried first in every young and active patient with SPSD. Majority of these patients can avoid demanding and expensive alternative treatment methods such as complex surgical procedures. This topical therapy method makes surgical SPSD a dermatologically treatable disease.
\end{abstract}

Key words: pilonidal sinus, polyphenols, topical, ultrasonography.

\section{Introduction}

Sacrococcygeal pilonidal sinus disease (SPSD) is a common and well-known entity which was described by Herbert Mayo in 1833. The term "pilonidal" (Latin: pilus = hair and nidus = nest) refers to a disease consisting of hair-containing sinuses in the sacrococcygeal area [1]. For many years the cause of SPSD has been controversial. Many authors proposed congenital origin of the lesion [2] but the acquired origin for SPSD proposed by Karydakis and Bascom is more commonly accepted today [3]. SPSD is a combination of symptoms with presenta- tions ranging from asymptomatic pits to painful draining lesions that are located in the sacrococcygeal area. It has also been reported in interdigital spaces in barbers [4], sheep shearers and dog groomers [5] in the literature. SPSD can be seen in both sexes but it is most commonly seen in hairy young males after puberty when sex hormones are known to affect pilosebaceous glands [6]. SPSD is associated with a requirement for hospital stay and causes a considerable loss of productivity, which is a very important consideration in young age population.

Although surgery is the primary treatment modality for SPSD, there is no general consensus of opinion re-

Address for correspondence: Assoc. Prof. Berna Aksoy MD, Department of Dermatology, Faculty of Medicine, Bahcesehir University, 34538 Istanbul, Turkey, phone: +90 5326665634, e-mail: bernaaaksoy@gmail.com

Received: 22.03.2018, accepted: 1.05.2018. 
garding one method [7] and none of surgical methods seems to be ideal. Complicated surgeries may cause poor wound healing, long hospital stay, patient discomfort, pain and even sepsis [8]. Acquired origin for SPSD pathogenesis is now accepted and there is always a risk of relapse following surgery especially in hairy male patients. So efforts to investigate more effective and less invasive treatment modalities continue. Other modalities for SPSD treatment include laser therapy, phenolization of the sinuses or depilation of buttocks by shaving, laser or creams [9]. However, phenolization needs an experienced physician and maximum attention to avoid complications. On the other hand, humic substances (natural polyphenols) are natural liquid biopolymers and are byproducts of soil organic matter degradation, which are present in our environment [10]. Polyphenols were shown to be effective in the treatment of SPSD [11]. Polyphenols

Table 1. Overall results of the study

\begin{tabular}{|c|c|}
\hline Parameter & $\begin{array}{l}n(\%) \text { or mean } \pm \text { SD } \\
\text { (min.-max./median) }\end{array}$ \\
\hline Age [years] & $30.6 \pm 7.1(18-45 / 30.5)$ \\
\hline \multicolumn{2}{|l|}{ Gender: } \\
\hline Male & $13(92.9)$ \\
\hline Female & $1(7.1)$ \\
\hline Total disease duration [years] & $3.0 \pm 3.7(0-14 / 2)$ \\
\hline Attack duration [days] & $86.0 \pm 85.2(1-270 / 60)$ \\
\hline \multicolumn{2}{|l|}{ Previous surgery: } \\
\hline None & $8(57.1)$ \\
\hline Abscess drainage & $2(14.3)$ \\
\hline Surgical SPSD treatment & $4(28.6)$ \\
\hline Cyst max diameter [mm] & $19.2 \pm 11.8(0-40 / 22)$ \\
\hline Cyst min diameter [mm] & $8.1 \pm 5.1(0-15 / 6.45)$ \\
\hline Treatment duration [months] & $3.8 \pm 2.1(1-10 / 3)$ \\
\hline \multicolumn{2}{|l|}{ Treatment usage: } \\
\hline Irregular & $3(21.4)$ \\
\hline Regular & $11(78.6)$ \\
\hline \multicolumn{2}{|l|}{ Treatment efficacy: } \\
\hline Non-effective & $1(7.1)$ \\
\hline Effective & $13(92.9)$ \\
\hline Recurrence & $3(21.4)$ \\
\hline Time to relapse [months] & $12.3 \pm 6.5(6-19 / 12)$ \\
\hline \multicolumn{2}{|l|}{ Re-treatment: } \\
\hline Polyphenols & $3(21.4)$ \\
\hline Follow-up period [months] & $18.3 \pm 13.5(2-52 / 19)$ \\
\hline \multicolumn{2}{|l|}{ Results: } \\
\hline SPSD free & $12(85.7)$ \\
\hline Active SPSD & $2(14.3)$ \\
\hline
\end{tabular}

are absorbed percutaneously and into pilonidal sinus tracts when they are applied topically over the skin [11]. A possible mechanism of action is removing microorganisms in the wound bed, antioxidant effects against reactive oxygen species, increase in the activity of lysosomes and chemotaxis, and promoting cytokines, interferons and tumour necrosis factor $\alpha$ (TNF- $\alpha)$ [11].

\section{Aim}

Ultrasonography was reported as an accurate method in the evaluation of sinus tracts and gives an idea about the full extent of the tract and its ramifications [12]. The aim of this study was to evaluate effectiveness of topical polyphenol treatment of SPSD by using physical examination and ultrasonographic assessment. An ultrasonographic examination verifies clinical findings and provides a high level of accuracy in the evaluation of treatment results.

\section{Material and methods}

Fourteen patients who were diagnosed with SPSD and treated with sodium humate $25 \%$ were included in this case series study retrospectively. Clinical evaluation and treatment of patients were performed in accordance with the ethical principles of the Declaration of Helsinki. Sodium humate $25 \%$ was used as the source of polyphenol. There were two natural polyphenol product forms used in the treatment of SPSD. The affected area was depilated before starting the treatment. The major form "DephytoPft Plus ${ }^{\oplus}$ lotion (Aventus Drug, Istanbul, Turkey)" was used before going to bed. A tea spoonful of DephytoPft Plus ${ }^{\circledast}$ lotion was poured onto the problematic region and the area was massaged for 2 to $3 \mathrm{~min}$. Later the area was covered with gauze and the gauze was left in place until morning. In the morning, the medication was washed out by Pilonol Gel ${ }^{\oplus}$ (Aventus Drug, Istanbul, Turkey). The treatment continued daily like this. All of the patients were advised to obey general personal hygiene rules and to keep the sacrococcygeal region depilated for at least 1 year. All of the patients were examined by superficial ultrasonography before and after treatment. Ultrasonographic evaluations were performed by Philips Ultrasound (Netherlands) by using $16 \mathrm{MHz}$ probe and Siemens Ultrasound VFX 13-5 (USA) by using 7.5 MHz thyroid probe. Demographic data, presence of sinuses and/or nodules clinically before and after treatment, recurrences, treatment durations, previous surgical treatments were retrieved from medical records.

\section{Statistical analysis}

SPSS 15.0 for Windows program was used for statistical analysis. Descriptive statistics were presented as number and percentage for categorical variables, and 
mean, standard deviation, minimum, maximum for numerical variables.

\section{Results}

Study results were summarized in Table 1 . Thirteen male patients and one female patient aged 18-45 years were admitted with the diagnosis of SPSD. Median disease duration was 2 years and median disease attack duration was 60 days. Six (42.9\%) patients had previous surgeries. The most common findings in physical examination were sinus openings and subcutaneous nodules at the pre-treatment clinical visit (Table 2). The most common findings in ultrasonography were abscess/ cyst and fistula at the pre-treatment visit (Table 2). All of the patients applied topical polyphenols for a mean 3.8 (min.-max.: 1-10) months; 78.6\% used the treatment regularly (Table 1 ). Treatment was effective in $92.9 \%$ but $3(21.4 \%)$ patients who got benefit from polyphenol therapy relapsed after a mean 12.3 (min.-max.: 6-19) months
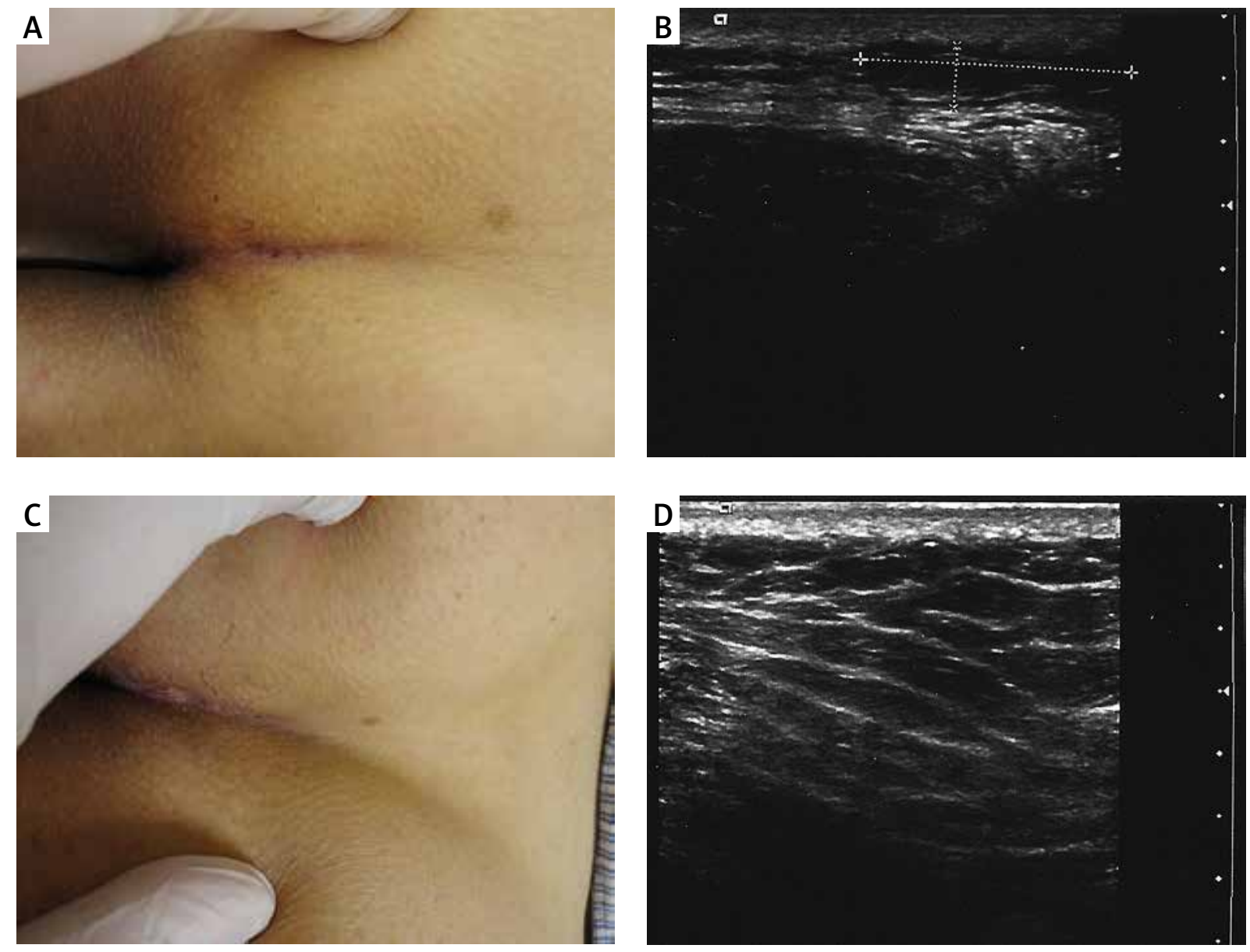

Figure 1. Case 4: a twenty-eight-year-old male patient suffered from SPSD for 2 years and experienced a disease attack lasting 30 days. Pre-treatment physical examination revealed sinus openings (some are erythematous) and left-hand-sided subcutaneous nodule (A) and ultrasonography revealed $21 \times 5 \mathrm{~mm}$ sized hypo-echoic area (fistula) in between superficial and deep fascia (B). He used topical polyphenols regularly for 3 months and control physical examination (C) and ultrasonography (D) were both normal. He had been followed up for 28 months without any recurrence 

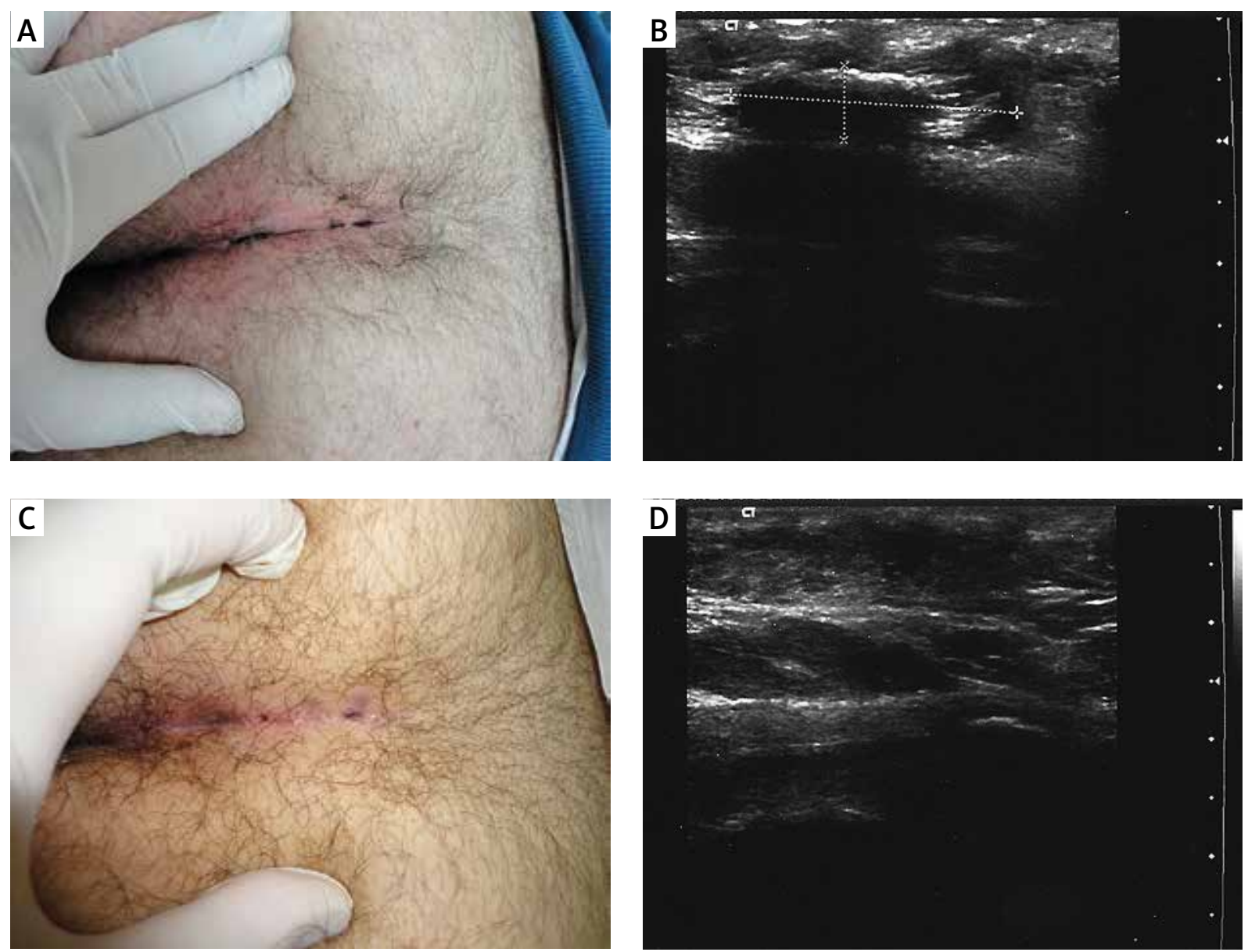

Figure 2. Case 12: a twenty-six-year-old male patient suffered from SPSD for 9 months. Pre-treatment physical examination revealed sinus openings, erythema, foul smelling mild drainage and small subcutaneous nodules (A) and ultrasonography revealed $23 \times 6 \mathrm{~mm}$ sized mass lesion with hypo- and hyper-echogenic areas (B). He used topical polyphenols regularly for 3.5 months and control physical examination revealed a few superficial sinus openings (C) and ultrasonographic findings were normal (D)

revealed that 12 (85.7\%) patients were disease-free and only 2 (14.3\%) patients had active SPSD (Table 1). Control physical examination and ultrasonography was completely normal in 11 patients without fistula, oedema, sinus openings or cysts (78.6\%) (Table 3).

\section{Discussion}

The search for new treatment alternatives for SPSD should continue as a considerable loss of productivity observed among affected young individuals causes a significant economic burden. Duman et al. [13] examined 19,013 students who attended state schools and determined that 1258 (6.6\%) students had SPSD in Turkey. In a recent study, it was reported that pilonidal cyst incidence increased among people who sat for long hours in front of their desks while working [14]. The ideal SPSD treatment method should be simple, decrease patient discomfort, prevent loss of workdays, shorten hospital stay and at the same time this method should have a low risk of complications and be associated with a low risk of recurrence.

In our study, patients used polyphenols topically over the affected area with massage. Nearly all of them $(n=13)$ got benefit from topical polyphenol treatment and 10 of them had complete remission. Only 3 patients experienced a recurrence and re-used the treatment. None of them indicated any significant side effects like irritation, erythema, burning and aching sensation. Long-term side effects of topical polyphenols have not been published yet but a study of blood, urine, and tissue levels of catechins following repeated treatments with systemic polyphenols in rat and mice models showed no side effects [15].

Nevertheless, the disadvantage of the topical polyphenol treatment is the need for regular topical applications between 1 and 10 months (mean: 3.8 months). Local skin reactions to topical medication may also adversely affect polyphenol therapy if they occur. Treatment results depend on patient compliance and some patients may request or require more rapid treatment alterna- 

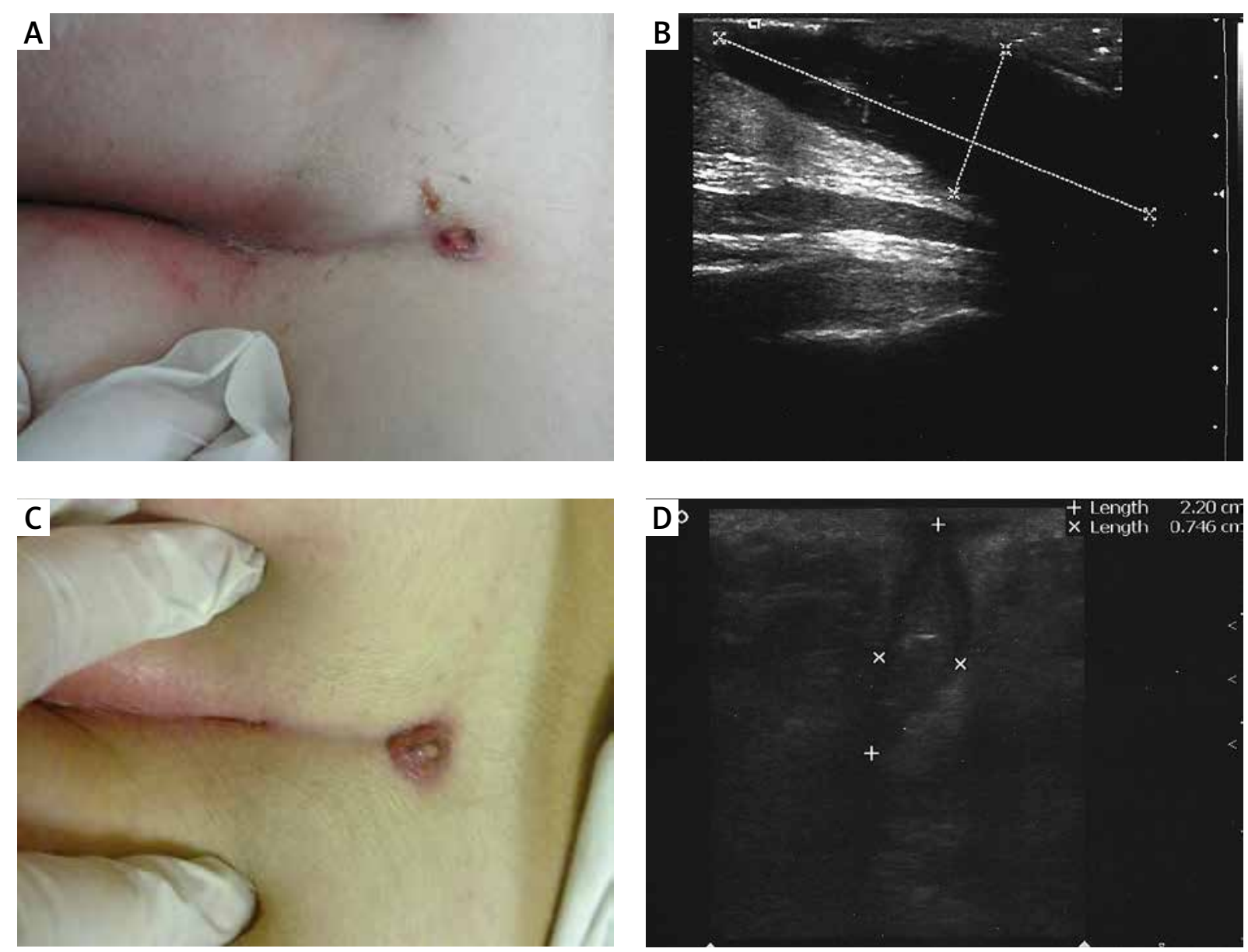

Figure 3. Case 8: an eighteen-year-old female patient suffered from SPSD for 2 months. Pre-treatment physical examination revealed erythematous cystic nodule with drainage and sinus openings (A) and ultrasonography revealed $40 \times 13 \mathrm{~mm}$ sized cystic mass lesion (B). She used topical polyphenols irregularly for 10 months. Her symptoms increased and decreased during treatment but she did not get complete benefit from topical polyphenol therapy. Control physical examination findings were similar to those of the initial physical examination (C) but post-treatment ultrasonography revealed $22 \times 7.5 \mathrm{~mm}$ sized blind ended sinus tract which decreased in size when compared to pre-treatment size. Ultrasonography also revealed presence of internal echogenicity and air within the sinus tract (D)

tives. Patients with complicated SPSD are not good candidates for topical polyphenol therapy.

Radical surgical excision of the lesion with primary wound closure or secondary wound healing is the most frequently used treatment for SPSD. Flaps were also used to cover soft tissue defects following radical excisions. Surgical site infection after radical excision is a frequent complication with a prevalence up to $24 \%$ [16]. Moreover, long hospital stay, analgesic intake, antibiotic use, postoperative care, duration of days off work and operative costs need to be taken into account. Any form of the radical surgical procedure is associated with an anatomical distortion in the natal cleft area and is associated with unsightly scars.

Another treatment option is phenol injection in the sinuses under local anaesthesia. This procedure is easy to perform when compared to surgical procedures and success rates of phenol injections and various surgical
Table 3. Post-treatment physical and ultrasonographic findings

\begin{tabular}{lll}
\hline Variable & $N$ & $\%$ \\
\hline
\end{tabular}

Control physical examination

findings:

\begin{tabular}{lcc}
\hline Normal & 11 & 78.6 \\
\hline Sinus openings & 3 & 21.4 \\
\hline Subcutaneous nodule & 1 & 7.1 \\
\hline Drainage & 1 & 7.1
\end{tabular}

Control USG findings:

\begin{tabular}{lcc}
\hline Normal & 11 & 78.6 \\
\hline Fistula & 2 & 14.3 \\
\hline Oedema & 1 & 7.1
\end{tabular}


procedures were reported to be similar. But this procedure is quite painful in the post-operative period and multiple sessions are generally necessary. The recurrence rate was reported to be between $7 \%$ and $27 \%$ [17]. Aksoy et al. [11] showed similar recurrence rates in topical polyphenol treatment. They reported that $53.1 \%$ of patients who experienced a relapse were treated again with topical polyphenols and similar treatment effectiveness was observed [11].

Dessily et al. [18] treated SPSD patients with a radial laser probe (FILAC ${ }^{\mathrm{TM}}$, Biolitec, Germany). The energy delivered to the lesions causes the destruction of the sinus epithelium and this leads to simultaneous obliteration of the tract. The success rate is good but the procedure needs to be done by an experienced physician and requires more expensive equipment and complications like hematomas and abscesses were reported. The cost of surgical treatment for SPSD was reported to be $£ 670$ for day-care patients and $£ 2400$ for in-patients [19]. In contrast, topical polyphenol treatment of SPSD costs approximately $£ 150$ for 3 months [11]. As the treatment duration increases, the treatment cost will increase.

In recent years, negative pressure wound care products have been investigated as one of the adjuvant therapy options for SPSD. The mechanism of action is to absorb exudate which accumulates in the wound, and to reduce the number of bacteria and tissue oedema. Negative pressure wound care products accelerate wound healing but there are relative contraindications to this procedure such as necrotic tissue formation with eschar on the wound, priority of debridement requirement, presence of an untreated osteomyelitis around the wound, cancer in the wound and development of a fistula towards an organ or body cavity [20].

Clinical palpation of the pilonidal sinus tract may underestimate the size of the lesion. We evaluated SPSD lesions with ultrasonography for a quantitative assessment before and after treatment. Ultrasonography was reported as an accurate method of evaluating sinus tracts caused by SPSD in the literature [12]. Borders of the SPSD lesions were determined by ultrasonographic examination and ultrasonography revealed that 12 out of 14 patients had complete healing and the other 2 patients had a decrease in the size of their lesions in our study.

Limitations of our study were a retrospective character of the study, small number of patients and short follow-up period. So future randomized, controlled clinical trials comparing topical polyphenol treatment with the other available SPSD treatment methods are needed.

\section{Conclusions}

Topical polyphenol treatment is a promising treatment alternative. This article emphasizes the usage of ultrasonography as an objective evaluation method to assess the effectiveness of topical polyphenols in the treatment of SPSD. It has many advantages when it is compared with other treatment modalities such as ease of use, low cost, no days off work, less pain and efficacy comparable to other treatment alternatives. We advise initial use of topical polyphenols in young and active patients with mild to moderate forms of SPSD. We think that majority of the patients treated with topical polyphenols can avoid demanding and expensive treatment methods like surgery. SPSD is accepted as an acquired surgically treatable disease so effective surgical treatment with a very low risk of relapse must change the anatomy of natal cleft and this causes anatomic disfigurement and healing time is long. Topical polyphenol treatment is non-invasive and can also be used to treat relapses so the need for significant surgical procedures will definitely decrease with the use of this treatment method. Topical polyphenol treatment creates the renaissance of a surgically treatable disorder into a dermatological disorder that can be topically treated by a lotion.

\section{Conflict of interest}

The authors declare no conflict of interest.

\section{References}

1. Chintapatla S, Safarani N, Kumar S, Haboubi N. Sacrococcygeal pilonidal sinus: historical review, pathological insight and surgical options. Tech Coloproctol 2003; 7: 3-8.

2. da Silva JH. Pilonidal cyst: cause and treatment. Dis Colon Rectum 2000; 43: 1146-56.

3. Sengul I, Sengul D, Mocan G. Axillary pilonidal sinus: a case report. N Am J Med Sci 2009; 1: 316-8.

4. Patey DH, Scarff RW. Pilonidal sinus in a barber's hand with observations on postanal pilonidal sinus. Lancet 1948; 2: 13.

5. Mohanna PN, Al-Sam SZ, Flemming AF. Subungual pilonidal sinus of the hand in a dog groomer. Br J Plast Surg 2001; 54: 176-8.

6. Søndenaa K, Andersen E, Nesvik I, Søreide JA. Patient characteristics and symptoms in chronic pilonidal sinus disease. Int J Colorectal Dis 1995; 10: 39-42.

7. Thompson MR, Senapati A, Kitchen P. Simple day-case surgery for pilonidal sinus disease. Br J Surg 2011; 98: 198-209.

8. Stewart A, Donoghue J, Mitten-Lewis S. Pilonidal sinus: healing rates, pain and embarrassment levels. J Wound Care 2008; 17: 468-70.

9. Kayaalp C, Aydin C. Review of phenol treatment in sacrococcygeal pilonidal disease. Tech Coloproctol 2009; 13: 189-93.

10. Scalbert A, Johnson IT, Saltmarsh M. Polyphenols: antioxidants and beyond. Am J Clin Nutr 2005; 81: 215S-7S.

11. Aksoy HM, Aksoy B, Egemen D. Effectiveness of topical use of natural polyphenols for the treatment of sacrococcygeal pilonidal sinus disease: a retrospective study including 192 patients. Eur J Dermatol 2010; 20: 476-81.

12. Mentes O, Oysul A, Harlak A, et al. Ultrasonography accurately evaluates the dimension and shape of the pilonidal sinus. Clinics (Sao Paulo) 2009; 64: 189-92.

13. Duman K, Gırgın M, Harlak A. Prevalence of sacrococcygeal pilonidal disease in Turkey. Asian J Surg 2017; 40: 434-7. 
14. Harlak A, Mentes O, Kilic S, et al. Sacrococcygeal pilonidal disease: analysis of previously proposed risk factors. Clinics (Sao Paulo) 2010; 65: 125-31.

15. Kim S, Lee MJ, Hong J, et al. Plasma and tissue levels of tea catechins in rats and mice during chronic consumption of green tea polyphenols. Nutr Cancer 2000; 37: 41-8.

16. Al-Khamis A, McCallum I, King PM, Bruce J. Healing by primary versus secondary intention after surgical treatment for pilonidal sinus. Cochrane Database Syst Rev 2010; 1: CD006213.

17. Topuz O, Sözen S, Tükenmez M, et al. Crystallized phenol treatment of pilonidal disease improves quality of life. Indian J Surg 2014; 76: 81-4.

18. Dessily M, Charara F, Ralea S, Allé JL. Pilonidal sinus destruction with a radial laser probe: technique and first Belgian experience. Acta Chir Belg 2017; 117: 164-8.

19. Abdul-Ghani AK, Abdul-Ghani AN, Ingham Clark CL. Day-care surgery for pilonidal sinus. Ann R Coll Surg Engl 2006; 88: 656-8.

20. Isik A, Idiz O, Firat D. Novel approaches in pilonidal sinus treatment. Prague Med Rep 2016; 117: 145-52. 